\title{
DIVISIBILITY GRAPH FOR SYMMETRIC AND ALTERNATING GROUPS
}

\author{
ADELEH ABDOLGHAFOURIAN AND MOHAMMAD A. IRANMANESH \\ Abstract. Let $X$ be a non-empty set of positive integers and $X^{*}=X \backslash\{1\}$. \\ The divisibility graph $D(X)$ has $X^{*}$ as the vertex set and there is an edge \\ connecting $a$ and $b$ with $a, b \in X^{*}$ whenever $a$ divides $b$ or $b$ divides $a$. Let \\ $X=\operatorname{cs}(G)$ be the set of conjugacy class sizes of a group $G$. In this case, we \\ denote $D(\operatorname{cs}(G))$ by $D(G)$. In this paper we will find the number of connected \\ components of $D(G)$ where $G$ is the symmetric group $S_{n}$ or is the alternating \\ group $A_{n}$.
}

\section{Introduction}

There are several graphs associated to various algebraic structures, especially finite groups, and many interesting results have been obtained recently, as for example, in [1, 5, 8, 14].

Let $X$ be a set of positive integers and $X^{*}=X \backslash\{1\}$. Mark L. Lewis in [13] introduced two graphs associate with $X$, the common divisor graph and the prime vertex graph. The common divisor graph $\Gamma(X)$ is a graph with vertex set $V(\Gamma(X))=X^{*}$, and edge set $E(\Gamma(X))=\{\{x, y\}: \operatorname{gcd}(x, y) \neq 1\}$. Note that $\operatorname{gcd}(x, y)$ denotes the greatest common divisor of $x, y$. The prime vertex graph $\Delta(X)$ is a graph with vertex set $V(\Delta(X))=\rho(X)=\bigcup_{x \in X} \pi(x)$, where $\pi(x)$ is the set of primes dividing $x$ and edge set $E(\Delta(G))=\{\{p, q\}: p q$ divides $x, x \in X\}$.

Praeger and the second author defined a bipartite graph $B(X)$ in [11] and elucidated the connection between these graphs. The bipartite divisor graph $B(X)$ is

2010 Mathematics Subject Classification. 05C25, $20 \mathrm{E} 45$.

Key words and phrases. Divisibility graph, Symmetric group, Alternating group, Diameter, Connected component.

Corresponding author: M. A. Iranmanesh. 
a graph with the vertex set $V(B(X))=\rho(X) \bigcup X^{*}$, and the edge set $E(B(X))=$ $\left\{\{p, x\}: p \in \rho(X), x \in X^{*}\right.$ and $p$ divides $\left.x\right\}$.

Recently A. R. Camina and R. D. Camina in [6] introduced a new directed graph (or simply a digraph) using the notion of divisibility of positive numbers. The $d i$ visibility digraph $\vec{D}(X)$ has $X^{*}$ as the vertex set and there is an arc connecting $(a, b)$ with $a, b \in X^{*}$ whenever $a$ divides $b$. It is clear that the digraph $\vec{D}(X)$ is not strongly connected (where by strongly connected digraph we mean a digraph such that there exists a directed path between any of two vertices). So it is important to find the number of connected components of its underlying graph. We denote the underlying graph of $\vec{D}(X)$ by $D(X)$. By the diameter of a graph $\Omega, \operatorname{diam}(\Omega)$, we mean the maximum diameter of its connected components.

For a finite group $G$ and $g \in G$, let $g^{G}=\left\{x^{-1} g x: x \in G\right\}$ be the conjugacy class of $g$ in $G$ and $\operatorname{cs}(G)=\left\{\left|g^{G}\right|: g \in G\right\}$ be the set of conjugacy class sizes of $G$. If $\delta$ is a permutation, then the cycle decomposition of $\delta$ is its expression as a product of disjoint cycles. It is interesting to investigate the properties of the prime vertex graph, the common divisor graph, the bipartite divisor graph and the divisibility graph when $X=\operatorname{cs}(G)$. In this case we denote $\Gamma(\operatorname{cs}(G)), \Delta(\operatorname{cs}(G))$, $B(\operatorname{cs}(G))$ and $D(\operatorname{cs}(G))$ by $\Gamma(G), \Delta(G), B(G)$ and $D(G)$ respectively. For properties of $\Gamma(G), \Delta(G)$ and $B(G)$ we refer to $[2,3,8,12$.

In this paper we investigate the graph $D(G)$ where $G$ is the symmetric group $S_{n}$ or the alternating group $A_{n}$. Note that two vertices $a, b$ of this graph are adjacent if either $a$ divides $b$ or $b$ divides $a$. Let $\delta \in S_{n}$. Suppose that there are $k_{i}$ cycles of length $m_{i}(1 \leq i \leq r)$ in the cycle decomposition of $\delta$, such that $m_{i} \neq 1$ and $m_{i} \neq m_{j}$, for $1 \leq i, j \leq r$, then we denote it by $\delta=\left[1^{t}, m_{1}^{k_{1}}, \ldots, m_{r}^{k_{r}}\right]$ where $t=n-\sum_{i=1}^{r} k_{i} m_{i}$. Also we denote the vertex corresponding to $\left|g^{G}\right|$ in $D(G)$ by $v_{g}$. Throughout the paper, $p$ is a prime number. 
In Section 2 we recall some basic lemmas and theorems which we need in the next sections. In Section 3 we will find the number of connected components of $D\left(S_{n}\right)$. The main theorem of this section is Theorem 10 In Section 4 we will find the number of connected components of $D\left(A_{n}\right)$. The main theorem of this section is Theorem 16,

\section{PRELIMINARIES}

In this section we recall some basic technical facts that we will use later. See [4, Chapter 13], 7, Chapter 4] or [9, p.131] for proofs and details.

Lemma 1. Suppose that $\delta=\left[1^{t}, m_{1}^{k_{1}}, \ldots, m_{r}^{k_{r}}\right]$ where $t=n-\sum_{i=1}^{r} k_{i} m_{i}$, then $\left|C_{S_{n}}(\delta)\right|=\left(\prod_{i=1}^{r} k_{i} ! m_{i}{ }^{k_{i}}\right) t !$

Lemma 2. Let $\delta \in A_{n}$, then there is an odd permutation in $C_{S_{n}}(\delta)$ if and only if $\left|C_{S_{n}}(\delta)\right|=2\left|C_{A_{n}}(\delta)\right|$.

Corollary 3. Let $\delta \in A_{n}$. Then either $\left|\delta^{S_{n}}\right|=\left|\delta^{A_{n}}\right|$ or $\left|\delta^{S_{n}}\right|=2\left|\delta^{A_{n}}\right|$.

Lemma 4. Let $\delta \in A_{n}$ fixes at least two points. Then $\left|C_{S_{n}}(\delta)\right|=2\left|C_{A_{n}}(\delta)\right|$ and $\left|\delta^{A_{n}}\right|=\left|\delta^{S_{n}}\right|$.

Lemma 5. Suppose that $\delta=\left[1^{t}, m_{1}^{k_{1}}, \ldots, m_{r}^{k_{r}}\right]$ and $t \leq 1$. If there exists $i$ such that $m_{i}$ is even or there exists $i$ such that $m_{i}$ is odd and $k_{i} \geq 2$, then $\left|C_{S_{n}}(\delta)\right|=$ $2\left|C_{A_{n}}(\delta)\right|$ and $\left|\delta^{A_{n}}\right|=\left|\delta^{S_{n}}\right|$.

By Lemma 4 and Lemma 5 we have the following corollary.

Corollary 6. If $\delta=\left[1^{t}, m_{1}^{1}, \ldots, m_{r}^{1}\right] \in A_{n}$, each $m_{i}$ is odd and $t \leq 1$, then we have $\left|C_{A_{n}}(\delta)\right|=\left|C_{S_{n}}(\delta)\right|$ and $\left|\delta^{A_{n}}\right|=\frac{1}{2}\left|\delta^{S_{n}}\right|$. For other cases, we have $\left|C_{A_{n}}(\delta)\right|=$ $\frac{1}{2}\left|C_{S_{n}}(\delta)\right|$ and $\left|\delta^{A_{n}}\right|=\left|\delta^{S_{n}}\right|$. 
Lemma 7. Let $x=\sum_{i=1}^{r} k_{i} m_{i}$, then $\prod_{i=1}^{r} k_{i} ! m_{i}{ }^{k_{i}}$ divides $x$ !. In addition if $m_{i} \geq 3$, for some $1 \leq i \leq r$, then $2 \prod_{i=1}^{r} k_{i} ! m_{i}{ }^{k_{i}}$ divides $x !$.

Proof. We prove this lemma by induction on $r$. Let $r=1$, then

$$
x !=\prod_{i=0}^{k_{1} m_{1}-1}\left(k_{1} m_{1}-i\right)=k_{1} ! m_{1}^{k_{1}} \prod_{\substack{i=1 \\ m_{1} \nmid i}}^{k_{1} m_{1}-1}\left(k_{1} m_{1}-i\right) .
$$

If $m_{1} \geq 3$, then 2 divides $\prod_{\substack{i=1 \\ m_{1} \nmid i}}^{k_{1} m_{1}-1}\left(k_{1} m_{1}-i\right)$. Therefore $2 k_{1} ! m_{1}^{k_{1}}$ divides $x !$.

Suppose that $r=t$. So $x=\sum_{i=1}^{t} k_{i} m_{i}=x^{\prime}+k_{t} m_{t}$ where $x^{\prime}=\sum_{i=1}^{t-1} k_{i} m_{i}$. Since $\left(\begin{array}{c}x \\ k_{t} m_{t}\end{array}\right) \in \mathbb{N}$, we conclude that $x^{\prime} !\left(k_{t} m_{t}\right)$ ! divides $x$ !. By induction hypothesis $\prod_{i=1}^{t-1} k_{i} ! m_{i}{ }^{k_{i}}$ divides $x^{\prime} !$ and $k_{t} ! m_{t}^{k_{t}}$ divides $\left(k_{t} m_{t}\right) !$. Let $m_{i} \geq 3$ for some $1 \leq i \leq r$. Then, without loss of generality, we may assume $m_{t} \geq 3$. So $2 k_{t} ! m_{t}^{k_{t}}$ divides $\left(k_{t} m_{t}\right) !$. Therefore $2 \prod_{i=1}^{t} k_{i} ! m_{i}{ }^{k_{i}}$ divides $x !$.

Remark 8. Let $G$ be a finite group and $x \in G$. It is clear that $C_{G}(x) \leq C_{G}\left(x^{m}\right)$ for every natural number $m$. So $\left|\left(x^{m}\right)^{G}\right|$ divides $\left|x^{G}\right|$. This means that $v_{x}$ is adjacent to $v_{x^{m}}$ in $D(G)$.

\section{DiVISIBILITY GRAPH FOR $S_{n}$}

In this section we investigate the number of connected components of $D\left(S_{n}\right)$. We will prove that $D\left(S_{n}\right)$ has at most two connected components. If it is disconnected, then one of its connected components is an isolated vertex, that is, a copy of $K_{1}$.

It is easy to see that both $D\left(S_{1}\right)$ and $D\left(S_{2}\right)$ are null graphs, that is, have no vertices. Also for $n=3,4$ and $5, D\left(S_{n}\right)$ has two connected components (see Figure 10.

Lemma 9. Let $1 \neq \delta \in S_{n}, n>2$ and $p \geq n-1$. Then $p$ divides $\left|C_{S_{n}}(\delta)\right|$ if and only if $\delta$ is a cycle of length $p$, that is, $\delta=[1, p]$ or $\delta=[p]$. 


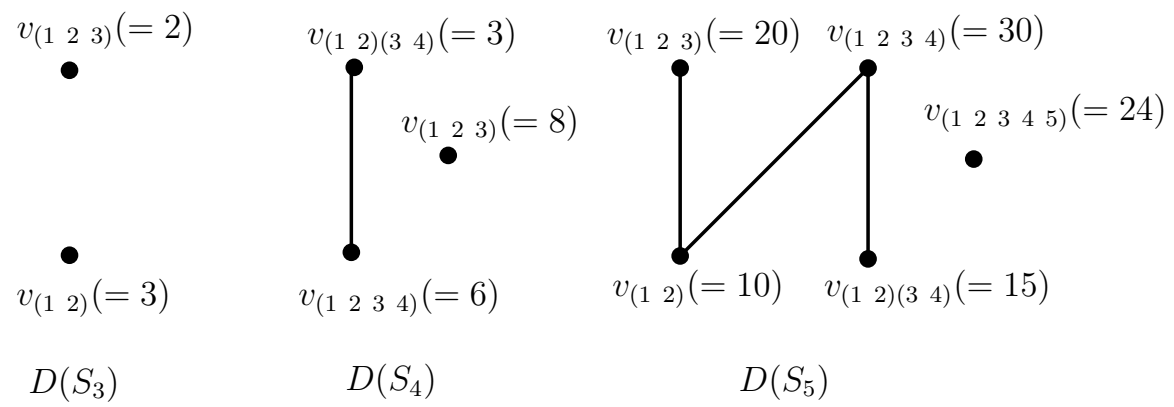

Figure 1. The graph $D\left(S_{n}\right)$ for $n=3,4$ and 5 .

Proof. First suppose $p$ divides $\left|C_{S_{n}}(\delta)\right|$. Assume, to the contrary, that $\delta=\left[1^{t}, m_{1}^{k_{1}}\right.$, $\left.\cdots, m_{r}^{k_{r}}\right]$ is not a $p$-cycle. By Lemma 1, $\left|C_{S_{n}}(\delta)\right|=\left(\prod_{i=1}^{r} k_{i} ! m_{i}{ }^{k_{i}}\right) t$ !. Since $p$ divides $\left|C_{S_{n}}(\delta)\right|$, then either $p$ divides $t$ ! or there exists $j$ such that $p$ divides $m_{j}$ or $k_{j}$ !. First assume that $p$ divides $m_{j}$. In this case we conclude that $p=m_{j}$. Hence $\delta$ is a cycle of length $p$ which is a contradiction. Now suppose that $p$ divides $k_{j} !$. Since $p$ is prime, we have $k_{j} \geq p \geq n-1$. Thus $k_{j} m_{j} \geq 2 n-2>n$, which is a contradiction too. Finally if $p$ divides $t$ !, then $t=n-\sum_{i=1}^{r} k_{i} m_{i} \geq p \geq n-1$, a contradiction.

For the other direction, note that if $\delta$ is a cycle of length $p$ then $\left|C_{S_{n}}(\delta)\right|=$ $p(n-p) !$

Theorem 10. Let $1 \neq \delta \in S_{n}$ and $n>6$. If $\delta$ is a $p$-cycle where $p \geq n-1$, then $v_{\delta}$ is an isolated vertex of $D\left(S_{n}\right)$. The other vertices are in a single connected component.

Proof. First suppose that $\delta$ is a cycle of length $p=n-i$ where $i \in\{0,1\}$. Assume, to the contrary, that $v_{\delta}$ has a neighbor, say $v_{\delta^{\prime}}$, where the cycle decomposition of $\delta^{\prime}$ is not similar to $\delta$ and $\left|C_{S_{n}}\left(\delta^{\prime}\right)\right|=x$. Then $n-i$ divides $x$, which is a contradiction by Lemma 9. Therefore in this case $v_{\delta}$ is an isolated vertex of $D\left(S_{n}\right)$.

Let $v_{\tau}$ be the vertex of $D\left(S_{n}\right)$ corresponding to an arbitrary transposition namely $\tau$. We prove that there exists a path between other arbitrary vertices of $D\left(S_{n}\right)$ and $v_{\tau}$ by using Lemma 1 and Lemma 7 . Since for every $\delta \in S_{n}$ there exists a natural 
number $m$ such that $\delta^{m}=\left[1^{t}, p^{t^{\prime}}\right]$, by Remark 8 it is enough to consider $\delta=\left[1^{t}, p^{t^{\prime}}\right]$.

So we have to consider three possible cases as follows:

(i) $\delta=\left[1^{n-2 k}, 2^{k}\right]$ and $k \geq 2$.

Let $\delta^{\prime}=\left[1^{n-2 k}, 4^{1}, 2^{(k-2)}\right]$,

$$
\frac{\left|C_{S_{n}}(\delta)\right|}{\left|C_{S_{n}}\left(\delta^{\prime}\right)\right|}=\frac{2^{k} k !(n-2 k) !}{4(k-2) ! 2^{k-2}(n-2 k) !}=k(k-1) \in \mathbb{N} .
$$

Since $\left|C_{S_{n}}\left(\delta^{\prime}\right)\right|$ divides $\left|C_{S_{n}}(\delta)\right|$, we conclude $\left|\delta^{S_{n}}\right|$ divides $\left|\delta^{\prime S_{n}}\right|$. Hence $v_{\delta}$ is adjacent to $v_{\delta^{\prime}}$. Also by Lemma 7 , there exists a positive integer $s$ such that

$$
\frac{\left|C_{S_{n}}(\tau)\right|}{\left|C_{S_{n}}\left(\delta^{\prime}\right)\right|}=\frac{2(n-2) !}{4(k-2) ! 2^{k-2}(n-2 k) !}=\frac{(n-2) !}{2^{k-1}(k-2) !(n-2 k) !}=\left(\begin{array}{c}
n-2 \\
2(k-1)
\end{array}\right) s \in \mathbb{N} .
$$

So $v_{\delta^{\prime}}$ is adjacent to $v_{\tau}$ and there is a path of length two between $v_{\delta}$ and $v_{\tau}$.

(ii) $\delta=\left[1^{n-k p}, p^{k}\right], p \neq 2$ and $k p \leq n-2$.

Let $\delta^{\prime}=(\alpha \beta) \delta$, where $\alpha$ and $\beta$ are two points fixed by $\delta$. So $\delta^{\prime}=\left[1^{n-k p-2}, 2^{1}, p^{k}\right]$ and we obtain

$$
\frac{\left|C_{S_{n}}(\delta)\right|}{\left|C_{S_{n}}\left(\delta^{\prime}\right)\right|}=\frac{k ! p^{k}(n-k p) !}{2 \cdot k ! p^{k} \cdot(n-k p-2) !}=\frac{(n-k p)(n-k p-1)}{2} \in \mathbb{N}
$$

Hence $v_{\delta}$ is adjacent to $v_{\delta^{\prime}}$. Also by Lemma 7 , there exists a positive integer $s$ such that

$$
\frac{\left|C_{S_{n}}(\tau)\right|}{\left|C_{S_{n}}\left(\delta^{\prime}\right)\right|}=\frac{2(n-2) !}{2 k ! p^{k}(n-k p-2) !}=\frac{(n-2) !}{k ! p^{k}(n-k p-2) !}=\left(\begin{array}{c}
n-2 \\
k p
\end{array}\right) s \in \mathbb{N}
$$

So there is a path of length 2 between $v_{\delta}$ and $v_{\tau}$.

(iii) $\delta=\left[1^{n-p k}, p^{k}\right], p \neq 2$ and $k p>n-2$.

In this case we have the following three subcases:

1) $k \geq 3$.

Let $\delta^{\prime}=\left[1^{n-p k},(2 p)^{1}, p^{(k-2)}\right]$,

$$
\frac{\left|C_{S_{n}}(\delta)\right|}{\left|C_{S_{n}}\left(\delta^{\prime}\right)\right|}=\frac{k ! p^{k}(n-k p) !}{2 p(k-2) ! p^{k-2}(n-k p) !}=\frac{p k(k-1)}{2} \in \mathbb{N} .
$$


Again we can conclude that $\left|\delta^{S_{n}}\right|$ divides $\left|\delta^{\prime S_{n}}\right|$ and so $v_{\delta}$ is adjacent to $v_{\delta^{\prime}}$. Since $p \neq 2$, we have $(k-1) p<k p-2$. Therefore $((k-1) p)$ ! divides $(k p-2)$ !. Hence by this fact and Lemma 7 , we can find positive integers $s$ and $s^{\prime}$ such that

$$
\begin{aligned}
\frac{\left|C_{S_{n}}(\tau)\right|}{\left|C_{S_{n}}\left(\delta^{\prime}\right)\right|} & =\frac{2(n-2) !}{2 p(k-2) ! p^{k-2}(n-k p) !}=\frac{(n-2) !}{(k-2) ! p^{k-1}(n-k p) !} \\
& =\frac{(k-1)(n-2) !}{(k-1) ! p^{k-1}(n-k p) !}=\frac{s(k-1)(n-2) !}{((k-1) p) !(n-k p) !}=\frac{s s^{\prime}(k-1)(n-2) !}{(k p-2) !(n-k p) !} \\
& =s s^{\prime}(k-1)\left(\begin{array}{c}
n-2 \\
k p-2
\end{array}\right) \in \mathbb{N} .
\end{aligned}
$$

This means that $v_{\delta^{\prime}}$ is adjacent to $v_{\tau}$ and there exists a path of length two between $v_{\delta}$ and $v_{\tau}$.

2) $k=2$.

Let $\delta^{\prime}=\left[1^{n-p}, p^{1}\right]$. Since $p>2$, there exists a positive integer $s$ such that

$$
\frac{\left|C_{S_{n}}\left(\delta^{\prime}\right)\right|}{\left|C_{S_{n}}(\delta)\right|}=\frac{p(n-p) !}{2 p^{2}(n-2 p) !}=\frac{(n-p) !}{2 p(n-2 p) !}=\left(\begin{array}{c}
n-p \\
p
\end{array}\right) s \in \mathbb{N}
$$

Thus we can conclude $\left|\delta^{\prime S_{n}}\right|$ divides $\left|\delta^{S_{n}}\right|$. Hence $v_{\delta}$ is adjacent to $v_{\delta^{\prime}}$. Also $p \leq n-2$, so by case(ii) there is a path of length two between $v_{\delta^{\prime}}$ and $v_{\tau}$.

3) $k=1$.

In this case $\delta$ is a $p$-cycle. So by Lemma $9 v_{\delta}$ is an isolated vertex.

Corollary 11. $D\left(S_{n}\right)$ has at most two connected components. If it is disconnected then one of its connected components is $K_{1}$.

Proof. We know that for $n \geq 6$, at most one of $n$ or $n-1$ is a prime. By Theorem 10 and Figure 1, we obtain the result.

\section{Divisibility GRAPH FOR $A_{n}$}

In this section we consider the divisibility graph for the alternating group $A_{n}$. We will show that $D\left(A_{n}\right)$ has at most three connected components and if it is 
disconnected then two of its connected components are $K_{1}$. We denote $\left|C_{A_{n}}(\delta)\right|=$ $\left(\frac{1}{2}\right)^{\sharp} x$ when we do not know whether $\left|C_{A_{n}}(\delta)\right|=\frac{1}{2} x$ or $\left|C_{A_{n}}(\delta)\right|=x$ for some $x \in \mathbb{N}$.

Remark 12. It is easy to see that $D\left(A_{1}\right), D\left(A_{2}\right)$ and $D\left(A_{3}\right)$ are null graphs. By using GAP [10] one can see that for $n=4,5,6,7$ and $8, D\left(A_{n}\right)$ has at most three connected components (see Figure Q).

In the rest of this section let $n \geq 9$.

Lemma 13. Let $1 \neq \delta \in A_{n}$ and $p \geq n-2$. Then $p$ divides $\left|C_{A_{n}}(\delta)\right|$ if and only if $\delta$ is a cycle of length $p$, that is, $\delta=\left[1^{2}, p\right], \delta=[1, p]$ or $\delta=[p]$.

Proof. First we assume that $p$ divides $\left|C_{A_{n}}(\delta)\right|$. Suppose, to the contrary, that $\delta=\left[1^{t}, m_{1}^{k_{1}}, \ldots, m_{r}^{k_{r}}\right]$ is not a $p$-cycle. By Lemma 1, Lemma 2 and Corollary 6 , $\left|C_{A_{n}}(\delta)\right|=\left(\frac{1}{2}\right)^{\sharp}\left(\prod_{i=1}^{r} k_{i} ! m_{i}{ }^{k_{i}}\right) t$ !. Since $p$ divides $\left|C_{A_{n}}(\delta)\right|$ we conclude that either $p$ divides $t$ ! or there exists $j$ such that $p$ divides $m_{j}$ or $k_{j}$ !. If $p$ divides either $m_{j}$ or $k_{j}$ !, then a similar argument to the proof of Lemma 9 shows that in this case either $p=m_{j}$ or $k_{j} m_{j} \geq 2 n-4>n$, which is a contradiction. If $p$ divides $t !=\left(n-\sum_{i=1}^{r} k_{i} m_{i}\right) !$, then $\sum_{i=1}^{r} k_{i} m_{i} \leq 2$. Hence $\delta$ should be a transposition, which is a contradiction too.

Now let $\delta$ be a cycle of length $p$. Note that $p$ is an odd number and every cycle of length $p$ is an even permutation. In this case $\left|C_{A_{n}}(\delta)\right|=\left(\frac{1}{2}\right)^{\sharp} p(n-p)$ !. Hence $p$ divides $\left|C_{A_{n}}(\delta)\right|$.

Before proving the main theorem of this section we shall prove two lemmas.

Lemma 14. Let $1 \neq \delta=\left[1^{t}, m_{1}^{k_{1}}, \ldots, m_{r}^{k_{r}}\right] \in A_{n}$. If there exists $j$ such that $k_{j}=1$ and $m_{j}=3$ then $v_{\delta}$ is adjacent to $v_{\left(\begin{array}{lll}1 & 2 & 3\end{array}\right)}$ in $D\left(A_{n}\right)$.

Proof. Without loss of generality we may assume that $k_{1}=1$ and $m_{1}=3$. Let $x=\sum_{i=1}^{r} k_{i} m_{i}$. By Corollary 6 and Lemma 7 , there exists a positive integer $s$ such 

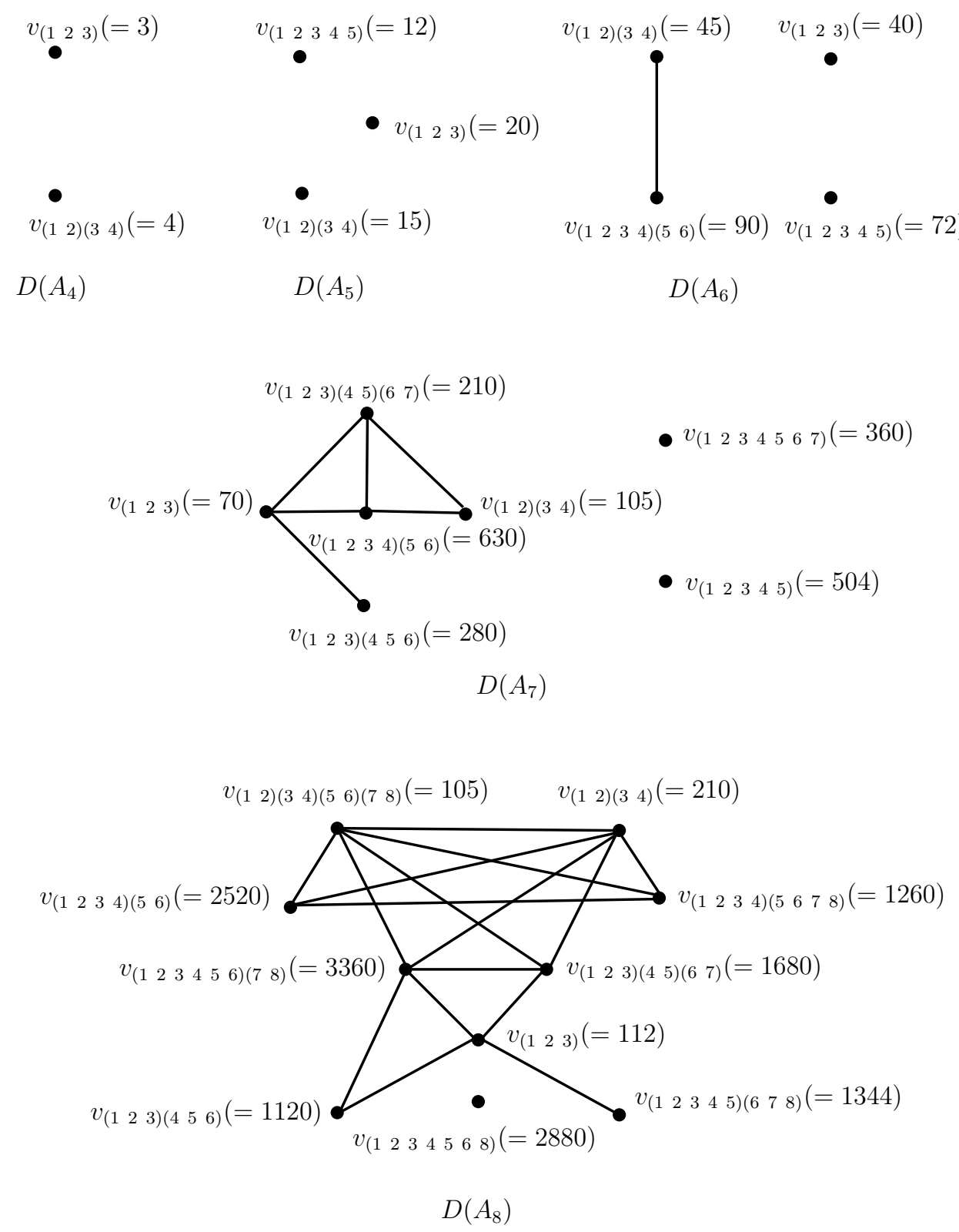

Figure 2. The graph $D\left(A_{n}\right)$ for $n=4,5,6,7$ and 8 .

that

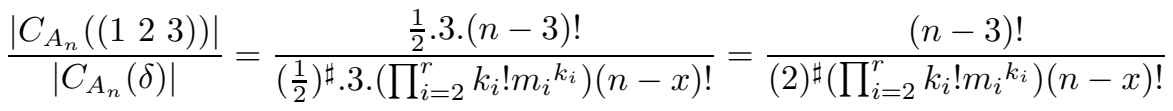

$$
\begin{aligned}
& =\left(\begin{array}{l}
n-3 \\
x-3
\end{array}\right) s \in \mathbb{N}
\end{aligned}
$$


Note that by Corollary 6, if 2 appears in denominator then we would have $t \leq 1$, $k_{i}=1$ and $m_{i}$ odd for $1 \leq i \leq r$. Since $n \geq 9$, there exists $i$ such that $m_{i} \geq 5$, so by Lemma 17, we can remove "2" from the denominator.

Thus $\left|C_{A_{n}}(\delta)\right|$ divides $\left|C_{A_{n}}\left(\left(\begin{array}{lll}1 & 2 & 3\end{array}\right)\right)\right|$. Therefore $\left|\left(\begin{array}{lll}1 & 2 & 3\end{array}\right)^{A_{n}}\right|$ divides $\left|\delta^{A_{n}}\right|$. So $v_{\delta}$ is adjacent to $v_{\left(\begin{array}{lll}1 & 2 & 3\end{array}\right)}$.

Lemma 15. Let $1 \neq \delta=\left[1^{t}, m_{1}^{k_{1}}, \ldots, m_{r}^{k_{r}}\right] \in A_{n}$. If $t \geq 3$ and for each $i, m_{i} \neq 3$ then there is a path of length two between $v_{\delta}$ and $v_{\left(\begin{array}{lll}1 & 2 & 3\end{array}\right)}$ in $D\left(A_{n}\right)$.

Proof. Let $\delta^{\prime}=(\alpha \beta \gamma) \delta$, where $\alpha, \beta$ and $\gamma$ are three points fixed by $\delta$. By Corollary 6 .

$$
\frac{\left|C_{A_{n}}(\delta)\right|}{\left|C_{A_{n}}\left(\delta^{\prime}\right)\right|}=\frac{\frac{1}{2}\left(\prod_{i=1}^{r} k_{i} ! m_{i}{ }^{k_{i}}\right) t !}{\left(\frac{1}{2}\right)^{\sharp} .3 \cdot\left(\prod_{i=1}^{r} k_{i} ! m_{i}{ }^{k_{i}}\right)(t-3) !}=\frac{t(t-1)(t-2)}{(2)^{\sharp} .3} \in \mathbb{N} .
$$

This implies that $\left|C_{A_{n}}\left(\delta^{\prime}\right)\right|$ divides $\left|C_{A_{n}}(\delta)\right|$ and hence $\left|\delta^{A_{n}}\right|$ divides $\left|\delta^{A_{n}}\right|$. So $v_{\delta}$

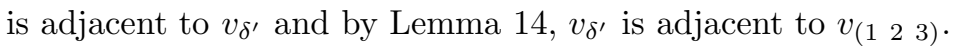

Theorem 16. Let $1 \neq \delta \in A_{n}$. If $\delta$ is a p-cycle where $p \geq n-2$ then $v_{\delta}$ is an isolated vertex of $D\left(A_{n}\right)$. The other vertices are in a single connected component.

Proof. First we show that if $\delta$ is a cycle of length $p$ where $p \geq n-2$, then $v_{\delta}$ is an isolated vertex. Let $p=n-i$ for $i \in\{0,1,2\}$. Then $\left|C_{A_{n}}(\delta)\right|=n-i$. Suppose $v_{\delta}$ has a neighbor say $v_{\delta^{\prime}}$, such that the cycle decomposition of $\delta^{\prime}$ is not the same as $\delta$. Let $\left|C_{A_{n}}\left(\delta^{\prime}\right)\right|=x$. In this case it is easy to see that $n-i$ divides $x$ which is impossible by Lemma 13

Now we are ready to show that the other vertices of $D\left(A_{n}\right)$ are all in the same connected component. We show that there exists a path between any other arbitrary vertex and the vertex corresponding to an arbitrary 3 -cycle namely $v_{\tau}$. By Lemma 1 and Corollary [6 $\left|C_{A_{n}}(\tau)\right|=\frac{1}{2} \cdot 3 .(n-3)$ !. We will use Lemma 1 . Corollary $\left[6\right.$ and Lemma 7 for our calculation. As for $S_{n}$, when $\delta \in A_{n}$ there is a 
natural number $m$ such that $\delta^{m}=\left[1^{t}, p^{t^{\prime}}\right]$, so by Remark 8 it is enough to consider $\delta=\left[1^{t}, p^{t^{\prime}}\right]$. There are the following three possible cases:

(i) $\delta=\left[1^{n-3 k}, 3^{k}\right]$ and $k \geq 2$.

If $k \geq 3$ then let $\delta^{\prime}=\left[1^{n-3 k}, 9^{1}, 3^{(k-3)}\right]$. Obviously $\delta^{\prime} \in A_{n}$ and we obtain

$$
\frac{\left|C_{A_{n}}(\delta)\right|}{\left|C_{A_{n}}\left(\delta^{\prime}\right)\right|}=\frac{\frac{1}{2} \cdot 3^{k} \cdot k !(n-3 k) !}{\left(\frac{1}{2}\right)^{\sharp} \cdot 9 \cdot\left(3^{k-3}\right)(k-3) !(n-3 k) !}=\frac{3 k(k-1)(k-2)}{(2)^{\sharp}} \in \mathbb{N} .
$$

Since $\left|C_{A_{n}}\left(\delta^{\prime}\right)\right|$ divides $\left|C_{A_{n}}(\delta)\right|$ we conclude $\left|\delta^{A_{n}}\right|$ divides $\left|\delta^{A_{n}}\right|$. So $v_{\delta}$ is adjacent to $v_{\delta^{\prime}}$. Also by Lemma 7 there exists a positive integer $s$ such that

$$
\begin{aligned}
\frac{\left|C_{A_{n}}(\tau)\right|}{\left|C_{A_{n}}\left(\delta^{\prime}\right)\right|} & =\frac{\frac{1}{2} \cdot 3 \cdot(n-3) !}{\left(\frac{1}{2}\right)^{\sharp} \cdot 9 \cdot\left(3^{k-3}\right)(k-3) !(n-3 k) !}=\frac{(n-3) !}{(2)^{\sharp} \cdot 3^{k-2}(k-3) !(n-3 k) !} \\
& =s\left(\begin{array}{c}
n-3 \\
n-3 k
\end{array}\right) \in \mathbb{N} .
\end{aligned}
$$

So $v_{\delta^{\prime}}$ is adjacent to $v_{\tau}$.

If $k=2$ then,

$$
\frac{\left|C_{A_{n}}(\tau)\right|}{\left|C_{A_{n}}(\delta)\right|}=\frac{3(n-3) !}{18(n-6) !}=\frac{(n-3)(n-4)(n-5)}{6} \in \mathbb{N}
$$

Again we can obtain $\left|\tau^{A_{n}}\right|$ divides $\left|\delta^{A_{n}}\right|$. So $v_{\delta}$ is adjacent to $v_{\tau}$.

(ii) $\delta=\left[1^{n-k p}, p^{k}\right], p \neq 3$ and $k>1$.

Note that if $k p \leq n-3$ then $\delta$ satisfies conditions of Lemma 15. So suppose $k p>n-3$.

We consider the following five subcases:

1) $k \geq 4$ and $p \neq 2$. In this case let $\delta^{\prime}=\left[1^{n-k p},(2 p)^{2}, p^{(k-4)}\right] \in A_{n}$ and $x=k p$.

$$
\frac{\left|C_{A_{n}}(\delta)\right|}{\left|C_{A_{n}}\left(\delta^{\prime}\right)\right|}=\frac{\frac{1}{2} k ! p^{k}(n-k p) !}{\frac{1}{2} \cdot 2 \cdot(2 p)^{2}(k-4) ! p^{k-4}(n-k p) !}=\frac{k ! p^{2}}{8(k-4) !} \in \mathbb{N}
$$

This yields that $\left|\delta^{A_{n}}\right|$ divides $\left|\delta^{A_{n}}\right|$. So $v_{\delta}$ is adjacent to $v_{\delta^{\prime}}$. 
Let $\delta^{\prime \prime}=\left[1^{n-2 p-3}, 3^{1}, p^{2}\right]$. By Lemma $[7$ and this fact that $p \geq 5$, we can find positive integers $s$ and $s^{\prime}$ such that

$$
\begin{aligned}
\frac{\left|C_{A_{n}}\left(\delta^{\prime \prime}\right)\right|}{\left|C_{A_{n}}\left(\delta^{\prime}\right)\right|} & =\frac{3(n-2 p-3) !}{4(k-4) ! p^{k-4}(n-k p) !}=\frac{3(n-2 p-3) ! s}{2(k p-4 p) !(n-k p) !} \\
& =\frac{3(n-2 p-3) ! s s^{\prime}}{(k p-2 p-3) !(n-k p) !}=3 s s^{\prime}\left(\begin{array}{c}
n-2 p-3 \\
k p-2 p-3
\end{array}\right) \in \mathbb{N} .
\end{aligned}
$$

So $v_{\delta^{\prime}}$ is adjacent to $v_{\delta^{\prime \prime}}$. Now $\delta^{\prime \prime}$ satisfies conditions of Lemma 14. Therefore there is a path of length three between $v_{\delta}$ and $v_{\tau}$.

2) $k>4$ and $p=2$. Since $\delta \in A_{n}$, in this case we must have $k \geq 6$. Let $\delta^{\prime}=$ $\left[1^{n-2 k},(2 k-2)^{1}, 2^{1}\right]$.

$$
\frac{\left|C_{A_{n}}(\delta)\right|}{\left|C_{A_{n}}\left(\delta^{\prime}\right)\right|}=\frac{\frac{1}{2} \cdot k ! 2^{k}(n-2 k) !}{\frac{1}{2} \cdot 2(2 k-2)(n-2 k) !} \in \mathbb{N} .
$$

So $v_{\delta}$ is adjacent to $v_{\delta^{\prime}}$. Also let $\delta^{\prime \prime}=\left[1^{n-7}, 2^{2}, 3^{1}\right]$.

$$
\frac{\left|C_{A_{n}}\left(\delta^{\prime \prime}\right)\right|}{\left|C_{A_{n}}\left(\delta^{\prime}\right)\right|}=\frac{8.3 .(n-7) !}{2(2 k-2)(n-2 k) !} \in \mathbb{N} .
$$

So $v_{\delta^{\prime}}$ is adjacent to $v_{\delta^{\prime \prime}}$. Now $v_{\delta^{\prime \prime}}$ satisfies conditions of Lemma 14, Therefore there is a path of length three between $v_{\delta}$ and $v_{\tau}$.

3) $k=4$ and $p=2$. Let $\delta^{\prime}=\left[1^{n-8}, 4^{2}\right]$.

$$
\frac{\left|C_{A_{n}}(\delta)\right|}{\left|C_{A_{n}}\left(\delta^{\prime}\right)\right|}=\frac{2^{4} .4 !(n-8) !}{4^{2} \cdot 2 !(n-8) !} \in \mathbb{N}
$$

This means $v_{\delta}$ is adjacent to $v_{\delta^{\prime}}$. Also let $\delta^{\prime \prime}=\left[1^{n-4}, 2^{2}\right]$.

$$
\frac{\left|C_{A_{n}}\left(\delta^{\prime \prime}\right)\right|}{\left|C_{A_{n}}\left(\delta^{\prime}\right)\right|}=\frac{2^{2} \cdot 2 !(n-4) !}{4^{2} \cdot 2 !(n-8) !} \in \mathbb{N} .
$$

So $v_{\delta^{\prime}}$ is adjacent to $v_{\delta^{\prime \prime}}$. By Lemma 15 there is a path of length two between $v_{\delta^{\prime \prime}}$ and $v_{\tau}$

4) $1<k<4$ and $p=2$. In this case we have $n-2 \leq k p \leq 6$ which is a contradiction with the assumption that $n \geq 9$. 
5) $1<k<4$ and $p \neq 2$. Since $p$ is odd, $\delta^{\prime}=\left[1^{n-k p+p}, p^{(k-1)}\right]$ is an even permutation.

In this case there exists a positive integer $s$ such that

$$
\frac{\left|C_{A_{n}}\left(\delta^{\prime}\right)\right|}{\left|C_{A_{n}}(\delta)\right|}=\frac{\frac{1}{2}(k-1) ! p^{k-1}(n-k p+p) !}{\frac{1}{2} k ! p^{k}(n-k p) !}=\frac{(n-k p+p) !}{k p(n-k p) !}=\left(\begin{array}{c}
n-k p+p \\
p
\end{array}\right) s \in \mathbb{N} .
$$

Therefore $\left|\delta^{\prime A_{n}}\right|$ divides $\left|\delta^{A_{n}}\right|$. So $v_{\delta}$ is adjacent to $v_{\delta^{\prime}}$. Again according to Lemma 15 there is a path of length two between $v_{\delta^{\prime}}$ and $v_{\tau}$.

(iii) $\delta=\left[1^{n-k p}, p^{k}\right], p \neq 3$ and $k=1$.

By Lemma 13 together with our earlier assumption $k p>n-3$, the vertex $v_{\delta}$ is isolated.

Corollary 17. $D\left(A_{n}\right)$ has at most three connected components. If it is disconnected, then two of its connected components are $K_{1}$.

Proof. We know that for any positive integer $n$, at most two of the positive integers $n, n-1$ and $n-2$ are primes. Hence by Theorem 16 and Remark 12, we obtain the result.

Remark 18. By using the fact that the distance between any vertices of $D\left(S_{n}\right)$ and $v_{\tau}$ is at most 4 (see proof of Theorem 10 and Remark 8) we can find that $\operatorname{diam}\left(D\left(S_{n}\right)\right) \leq 8$. A similar argument and using the proof of Theorem 16, shows that $\operatorname{diam}\left(D\left(A_{n}\right)\right) \leq 10$.

By considering $D\left(S_{n}\right)$ and $D\left(A_{n}\right)$ for some values of $n$ we may pose the following conjecture.

Conjecture 1. The best upper bound for the diameter of $D\left(S_{n}\right)$ and $D\left(A_{n}\right)$ is 4 .

\section{ACKNOWLEDGMENT}

The authors would like to thank the anonymous referee for helpful comments which improved the quality of this paper. 


\section{REFERENCES}

1. Abdollahi, A., Mohammadi Hassanabadi, A. (2007). Noncyclic Graph of a Group. Comm. Algebra $35: 2057-2081$.

2. Bertram, E. A., Herzego, M., Mann, A. (1990). On a graph related to conjugacy classes of groups. Bull. London Math. Soc. 22:569-575.

3. Bubboloni, D., Dolfi, S., Iranmanesh, M. A., Praeger, C. E. (2009). On bipartite divisor graphs for group conjugacy class sizes. J. Pure Appl. Algebra 213:1722-1734.

4. Cameron, P. (1994). Combinatorics: Topics, Techniques, Algorithmes. Cambridge: Cambridge Univ. Press.

5. Cameron, P., Ghosh, S. (2011). The power graph of a finite group. Discrete Math. 311:12201222.

6. Camina, A. R., Camina, R. D. (2011). The influence of conjugacy class sizes on the structure of finite groups: a survey. Asian-Eur. J. Math. 4:559-588.

7. Camina, A., Lewis,B. (2011). An Introduction to Enumeration. London: Springer-Verlag.

8. Casolo, C., Dolfi, S. (1996). The diameter of a conjugacy class graph of finite groups. Bull. London Math. Soc. 28:141-148.

9. Dummit, D. S., Foote, R. M. (2004). Abstract Algebra. The third edition. New Jersey: Wiley.

10. The GAP - Groups, Algorithms and Programming. Version 4.4.12, 2008. www.gap-system.org".

11. Iranmanesh, M. A., Praeger, C. E. (2010). Bipartite divisor graphs for integer subsets. Graphs Combin. 26:95-105.

12. Kazarin, L. S. (1981). On groups with isolated conjugacy classes. IzV. Vyssh. UchebnZaved. mat. 25:40-45.

13. Lewis, M. L. (2008). An overview of graphs associated with character degrees and conjugacy class sizes in finite groups. Rocky Mountain J. Math. 38:175-211.

14. Wang, L., Shi, W. (2008). A New Characterization of $A_{10}$ by its Noncommuting Graph. Comm. Algebra 36:523-528.

A. Abdolghafourian, Department of Mathematics

YAZD University, YAZD, 89195-741, IRAN

E-mail address: A.Abdolghafourian@stu.yazd.ac.ir

M. A. Iranmanesh, Department of Mathematics

YAZD UNIVERSITY, YAZD, 89195-741, IRAN

E-mail address: iranmanesh@yazd.ac.ir 